\title{
Optimal Structure $(k)$ Designs for Comparing Test Treatments with a Control
}

\author{
M. A. Chowdhury \\ Department of Statistics, University of Manitoba \\ Winnipeg, MB, R3T 2N2, Canada \\ umchowd6@myumanitoba.ca \\ S. Mandal \\ Department of Statistics, University of Manitoba \\ Winnipeg, MB, R3T 2N2, Canada \\ saumen.mandal@umanitoba.ca \\ D. K. Ghosh \\ Department of Statistics, Saurashtra University \\ UGC BSR Faculty Fellow, Rajkot - 360005, India \\ ghosh_dkg@rediffmail.com \\ S. C. Bagui \\ Department of Mathematics and Statistics, University of West Florida \\ Pensacola, FL 32514, USA \\ sbagui@uwf.edu
}

Received 29 October 2015

Accepted 15 December 2016

\begin{abstract}
Mukerjee (1979) introduced structure $(k)$ property of a factorial design. In this article, we introduce structure $\left(k_{1}\right)$, structure $\left(k_{2}\right)$ and structure $\left(k_{1} k_{2}\right)$ properties of a factorial design. We establish properties of each of these structure designs in terms of the incidence and characteristic matrices of the designs. Furthermore, we develop methods of obtaining optimal $R$-type structure $(k)$ designs and show that such designs are trace, $A$ - and $M V$-optimal. The proposed methodologies are easy to follow and the construction of the designs comes out in a simple form.
\end{abstract}

Keywords: Factorial design; hypercubic design; $R$-type structure designs; $A$-optimality; $M V$-optimality; trace optimality.

2000 Mathematics Subject Classification: 62K15, 62K05

Copyright (C) 2017, the Authors. Published by Atlantis Press.

This is an open access article under the CC BY-NC license (http://creativecommons.org/licenses/by-nc/4.0/). 


\section{Introduction}

Consider a factorial experiment with $m$ factors such that $i$ th factor has $s_{i}$ levels for $i=1,2, \ldots, m$. Therefore the total number of treatment combinations in the experiment is $v=\prod_{i=1}^{m} s_{i}$. Let $N=\left(n_{i j}\right)$ $(i=1,2, \ldots, v ; j=1,2, \ldots, b)$ be the incidence matrix of a block design, where $n_{i j}=1$ or 0 if the $i$ th treatment occurs in the $j$ th block or absent in the $j$ th block respectively. The calculus for factorial arrangements has been applied to the analysis of several classes of experimental designs and has been addressed by several authors such as Kurkjian and Zelen [7, 8], Zelen and Federer [18, 19], Paik and Federer [11], Mukerjee [9, 10] and Cotter [1]. Kurkjian and Zelen [7] applied the calculus for factorial arrangements to the analysis of block designs. They showed that the concurrence matrix $N N^{\prime}$ of the design with an incidence matrix $N_{(v \times b)}$ can be expressed as a linear combination of Kronecker products $(\otimes)$ of $I_{i}$ and $E_{i}$ matrices, where $I_{i}$ is an identity matrix of order $s_{i}$ and $E_{i}$ is a $s_{i} \times s_{i}$ matrix with each element unity. That is, $N N^{\prime}$ satisfies the property

$$
N N^{\prime}=\sum_{s=0}^{m}\left\{\sum_{\delta_{1}+\delta_{2}+\ldots+\delta_{m}=s} h\left(\delta_{1}, \delta_{2}, \ldots, \delta_{m}\right)\left(D_{1}^{\delta_{1}} \otimes D_{2}^{\delta_{2}} \otimes \ldots \otimes D_{m}^{\delta_{m}}\right)\right\}
$$

where $\delta_{i}=0$ or $1, h\left(\delta_{1}, \delta_{2}, \ldots, \delta_{m}\right)$ are constants depending on $\delta_{i}$, and $D_{i}^{\delta_{i}}$ is a $s_{i} \times s_{i}$ matrix defined by

$$
D_{i}^{\delta_{i}}= \begin{cases}I_{i} & \text { if } \delta_{i}=0 \\ E_{i} & \text { if } \delta_{i}=1\end{cases}
$$

A design satisfying (1.1) is called a property $(A)$ design. The class of designs that have property $(A)$ includes many designs that are used in practice such as randomized block designs, balanced incomplete block designs, group divisible designs and bulk of the Kronecker designs constructed by Vartak [17], Shah [13] and Rao [12]. Let $C$ and $C^{+}$denote the characteristic matrix of the design and Moore-Penrose inverse of $C$ respectively. Since $C^{+}$is the Moore-Penrose inverse of $C$, it must satisfy the conditions $C C^{+} C=C$ and $C^{+} C C^{+}=C^{+}$. Note that if $N N^{\prime}$ satisfies (1.1), i.e., if $N N^{\prime}$ has property $(A)$, then the characteristic matrices $C$ and $C^{+}$also have this property. This class of designs is particularly suitable for use in asymmetrical factorial experiments. Also, the analysis of the designs is simple and elegant even if there is no factorial structure underlying the treatment combinations.

Sia [14] studied property $(A)$ designs with respect to the $A$-optimality criterion. Zelen and Federer [19] extended the idea of property $(A)$ design to row-column designs. If the column incidence matrix $N_{(v \times b)}$ satisfies (1.1), it is still called a property $(A)$ design. However, if the row incidence matrix $\tilde{N}_{(v \times b)}$ satisfies a similar property, then the design is called a property $(B)$ design. Designs in which the row and column incidence matrices satisfy (1.1) are termed as property $(A B)$ designs. Zelen and Federer [19] derived the intra-block analysis for property $(A B)$ designs. Paik and Federer [11] showed that the property $(A)$ design and property $(B)$ design implies property $(A B)$ design.

Mukerjee [10] noted a major limitation of the previous work on factorial structure. The limitations are that the results are given in terms of a generalized inverse of $C$-matrix. Results in terms of generalized inverse of $C$-matrix are provided by Cotter [1], and John and Smith [6]. In fact, Mukerjee [10] introduced the notion of structure $(k)$ design and determined a simple set of necessary and sufficient conditions for factorial structure which can be stated in terms of the $C$ matrix. In this context, Mukerjee [10] considered that for any vector $x=\left(x_{1}, x_{2}, \ldots, x_{m}\right), x_{i}=0,1$; 
$W^{x}=W_{1}^{x_{1}} \times W_{2}^{x_{2}} \times \ldots \times W_{m}^{x_{m}}$, where $W_{i}=I_{i}-s_{i}^{-1} E_{i}, i=1,2, \ldots, m$. We will discuss some properties of structure $(k)$ design in terms of $W^{x}$ and a permutation matrix later on. On the basis of Mukerjee [10] we define a property of a design and call it a structure $(k)$ design.

A $v \times v$ matrix $D$, where $v=\prod_{i=1}^{m} s_{i}, s_{i} \geq 2$ for all $i$, is said to have structure $(k)$, if $D$ can be expressed as a linear combination of Kronecker products of permutation matrices of order $s_{1}, s_{2}, \ldots, s_{m}$ (taken in that order), i.e., if $D$ can be written as

$$
D=\sum_{j=1}^{w} r_{j}\left(R_{j 1} \otimes R_{j 2} \otimes \ldots \otimes R_{j m}\right)
$$

where $w$ is some positive integer, $r_{1}, r_{2}, \ldots, r_{w}$ are some numbers, and for each $j, R_{j i}$ is a $s_{i} \times s_{i}$ permutation matrix.

Clearly property $(A)$ design is a special case of structure $(k)$ design. The structure $(k)$ property can be expressed in terms of $N N^{\prime}$ or $C$-matrix.

In the present work, we introduce structure $\left(k_{1}\right)$, structure $\left(k_{2}\right)$ and structure $\left(k_{1} k_{2}\right)$ properties of a factorial design, and show that structure $\left(k_{1}\right)$ design and structure $\left(k_{2}\right)$ design implies structure $\left(k_{1} k_{2}\right)$ design using the properties of the incidence and characteristic matrices of the designs. We also study the structure $(k)$ designs with respect to the trace, $A$ - and $M V$-optimality criteria. Starting from a structure $(k)$ design and augmenting one control in each block, we develop methods of obtaining optimal $R$-type structure $(k)$ designs and show that such designs are trace, $A$ - and $M V$ optimal. The proposed methodologies are easy to follow and the construction of the designs comes out in a simple form.

\section{Preliminaries}

Definition 2.1. Proper Matrix: A square matrix where all row sums and column sums are equal is called a proper matrix.

Definition 2.2. Permutation Matrix: A square matrix with non-negative entries in which all row sums and column sums are equal to unity is called a permutation matrix.

Lemma 2.1. Any proper matrix can be expressed as a linear combination of permutation matrices of the same order.

Lemma 2.2. For any $v \times v$ permutation matrix $R$ and for any $x, W^{x} R W^{x}$ has structure $(k)$.

Lemma 2.3. A $v \times v$ matrix $A$, where $v=\prod_{i=1}^{m} s_{i}, s_{i} \geq 2$ for all $i$, has structure $(k)$ if and only if $A$ is expressible as a linear combination of Kronecker products of proper matrices of order $s_{1}, s_{2}, \ldots, s_{m}$ (taken in that order).

Lemma 2.4. For a connected block design, a necessary and sufficient condition for factorial structure is that column $C$-matrix has structure $(k)$.

The above lemmas are due to Mukerjee [10]. Below we state a lemma which is due to Jacroux [5].

Lemma 2.5. Let $d\left(v^{\prime}, b, k^{\prime}\right)$ be the semi-rectangular (SR) design obtained by reinforcing each block with a control treatment of Group Divisible (GD) design $\bar{d}$ having parameters $v=m n, b, r=b k / v$, $m=2, n=v / 2$ and $\lambda_{2}=\lambda_{1}+1$, then design $d$ is trace optimal.

Theorem 2.1. There exists a hypercubic design (HCD), $\bar{d}$, having parameters $v=t^{m}(t=2), r=$ $\left(\begin{array}{c}m+n-1 \\ h+n-1\end{array}\right), n=k / t^{h}=1, b=v r / k, k=t^{h}(h=1,2, \ldots, m-1), \lambda_{i}=\left(\begin{array}{c}m-i \\ h-i\end{array}\right)$, for $i \leq h$ and $\lambda_{j}=0$ for $j>h$ if $\bar{d}$ satisfies the following conditions: 
(i) the size of the $i$ th group $G_{i}(i=1,2, \ldots, k)$ should be a multiple of block size $k$

(ii) the block size $(k)$ should be an even number

(iii) the $i$ th group $G_{i}$ contains $v / k$ treatment combinations

(iv) each treatment combination occurs once and only once in each group $G_{i}(i=1,2, \ldots, k)$.

Theorem 2.2. There exists a hypercubic design (HCD), $\bar{d}$, having parameters $v=t^{m}(t=3)$, $r=2^{m-k+2}, b=v r / k, k=n$ for $1<n \leq m+1$, and

$$
\lambda_{i}= \begin{cases}0 & \text { if } i \leq m-1 \\ 1 & \text { otherwise }\end{cases}
$$

if $\bar{d}$ satisfies the following conditions:

(i) the block size $(k)$ is an integer, that is, $k=n$,

(ii) the size of the $i$ th group $G_{i}$ should be a multiple of $t^{n}$ for $i=1,2, \ldots, t^{h-1}, h=1,2, \ldots, m-1$,

(iii) the levels of the factor at $i$ th position are not the same for all the factors while taking $k$ treatment combinations from $G_{i}$ different groups and keeping them in one block.

Theorem 2.1 and 2.2 are useful for the construction of SR-HCD designs.

Theorem 2.3. Let $d\left(v^{\prime}, b, k^{\prime}\right)$ be a SR-HCD design obtained by reinforcing each block with a control treatment of hypercubic design, $\bar{d}$, having parameters same as in Theorems 2.1 and 2.2. If $d$ satisfies the conditions discussed in Lemma 2.5, then $d$ is trace optimal.

Theorem 2.4. Let $r_{0}$ be the number of replication of the control treatment in $d^{*}$, where $d^{*} \in D_{r_{0}}(v+$ $1, b, k)$ is a Group Divisible Treatment Design (GDTD) having parameters $m=2, n=v / 2$, and $\lambda_{2}=\lambda_{1}+1$. If $m_{1}\left(r_{0}\right) \leq m_{2}\left(r_{0}\right)$ and $d^{*}$ is such that $\operatorname{trC}_{d^{*} 11}^{-1} \leq H_{2}\left(r_{0}\right)$, then $d^{*}$ is $A$-optimal in $D_{r_{0}}(v+1, b, k)$, where $C_{d^{*} 11}$ is the principal submatrix obtained from $C_{d}^{*}$ after deleting row 1 and column 1 and $H_{2}\left(r_{0}\right)=\left(1 / m_{1}\left(r_{0}\right)\right)+\left((v-1) / m_{4}\left(r_{0}\right)\right)$ with $m_{1}\left(r_{0}\right)=b(k-1) / v k, m_{4}\left(r_{0}\right)=\{A-$ $\left.(2 / k)-m_{1}\left(r_{0}\right)\right\} /(v-1), A=b(k-1)^{2} / k$.

Theorem 2.5. For a given value of $r_{0}$, let $d^{*} \in D_{r_{0}}(v+1, b, k)$ be a Group Divisible Treatment Design $(\operatorname{GDTD}(s+1))$ such that

$$
\begin{gathered}
r_{d^{*} 0} k-\lambda_{d^{*} 00}=r_{0} k-\lambda\left(r_{0}\right), \bar{\lambda}_{0}=\left(r_{0} k-\lambda\left(r_{0}\right)\right) / v, \\
r_{d^{*} i}-\lambda_{d^{*} i i}=R\left(r_{0}\right)(k-1), \text { for } i=1, \ldots, v, \\
\bar{\lambda}_{2}=\bar{\lambda}_{1}+1 \text { where } \bar{\lambda}_{1}=\left[\left(R\left(r_{0}\right)(k-1)-\bar{\lambda}_{0}\right) /(v-1)\right] .
\end{gathered}
$$

Also, for positive integers $p$ and $q$, define $B(p, q)=\left(1-((1-\bar{m} p)(1-\bar{m} q))^{1 / 2}\right) / \bar{m}$ and let $\bar{m} k=\left(k / v \bar{\lambda}_{0}\right)+\left((\bar{v}-1) s k / v\left(\bar{v}(s-1) \bar{\lambda}_{2}+v \bar{\lambda}_{1}+\bar{\lambda}_{0}\right)\right)+\left((s-1) k / v\left(v \bar{\lambda}_{2}+\bar{\lambda}_{0}\right)\right)$ where $\bar{v}=v / s$. Now, if

(1) $r_{0} k-\lambda\left(r_{0}\right)-2<v B\left(r_{0} k-\lambda\left(r_{0}\right)-2, R\left(r_{0}\right)(k-1)\right)$

(2) $\left(R\left(r_{0}\right)-1\right)(k-1)$ or $r_{0} k-\lambda\left(r_{0}\right)$ satisfies one of the appropriate inequalities $1 /\left(R\left(r_{0}\right)-\right.$ 1) $(k-1)>\bar{m}$ or $r_{0} k-\lambda\left(r_{0}\right)<(v-2) B\left(r_{0} k-\lambda\left(r_{0}\right), R\left(r_{0}\right)(k-1)\right)+B\left(r_{0} k-\lambda\left(r_{0}\right),\left(R\left(r_{0}\right)-\right.\right.$ $1)(k-1))+B\left(r_{0} k-\lambda\left(r_{0}\right),\left(R\left(r_{0}\right)+1\right)(k-1)\right)$

(3) $\bar{m} k<\min \left\{\left(\bar{c}_{d 00}+\bar{c}_{d i i}+2 \bar{c}_{d i 0}\right) /\left(\bar{c}_{d 00} \bar{c}_{d i i}-\bar{c}_{d i o}^{2}\right),\left(\bar{c}_{d 00}+\bar{c}_{d j j}+2 \bar{c}_{d j 0}\right) /\left(\bar{c}_{d 00} \bar{c}_{d j j}-\bar{c}_{d j 0}^{2}\right)\right\}$

(4) $\bar{m} k<\left\{\bar{c}_{d p p}\left(\bar{c}_{d 00}+\bar{c}_{d q q}+2 \bar{c}_{d p q}\right)-\left(\bar{c}_{d p 0}+\bar{c}_{d p q}\right)^{2}\right\} /\left\{\bar{c}_{d 00} \bar{c}_{d p p} \bar{c}_{d q q}-\bar{c}_{d 00} \bar{c}_{d p q}^{2}-\bar{c}_{d p p} \bar{c}_{d q 0}^{2}-\right.$ $\left.\bar{c}_{d q q} \bar{c}_{d p 0}^{2}+2 \bar{c}_{d p 0} \bar{c}_{d q 0} \bar{c}_{d p q}\right\}$

then $d^{*}$ is MV-optimal in $D_{r_{0}}(v+1, b, k)$, where $N\left(r_{0}\right)=\left[\frac{r_{0}}{b}\right], \lambda\left(r_{0}\right)=\left(r_{0}-b N\left(r_{0}\right)\right)\left(N\left(r_{0}\right)+\right.$ $\left.1)^{2}+\left(b-r_{0}+b N\left(r_{0}\right)\right) N^{2}\left(r_{0}\right), R\left(r_{0}\right)=\left[\left(b k-r_{0}\right) / v\right)\right], r_{d^{*} i}$ is the $i^{\text {th }}$ row sum of $N_{d^{*}}$ (incidence matrix) which represent the number of times treatment $i$ is replicated in the design $d^{*}$ and $\lambda_{d^{*} i i}$ is 
the diagonal entries of $i$ th row and $i$ th column of the concurrence matrix $N_{d^{*}} N_{d^{*}}^{\prime}$ for $i=1, \ldots, v$, $\bar{c}_{d 00}=\left(r_{0} k-\lambda\left(r_{0}\right)\right) / k, \bar{c}_{d i i}=R\left(r_{0}\right)(k-1) / k, \bar{c}_{d i o}=-\left(\bar{\lambda}_{0}-1\right) / k, \bar{c}_{d j j}=\left(R\left(r_{0}\right)(k-1)-2\right) / 2$, $\bar{c}_{d j 0}=-\bar{\lambda}_{0} / k, \bar{c}_{d p p}=\bar{c}_{d q q}=R\left(r_{0}\right)(k-1) / k, \bar{c}_{d p 0}=\bar{c}_{d q 0}=-\bar{\lambda}_{0} / k,-\bar{c}_{d p q}=\left(\bar{\lambda}_{1}-1\right) / k$ or $\left(\bar{\lambda}_{1}+2\right) / k$, and [.] denotes the greatest integer function.

For further details see Jacroux [2 - 4]. Theorems 2.1, 2.2, 2.3 are due to Thannipara [15]. Theorem 2.4 is due to Jacroux [2]. Theorem 2.5 is due to Jacroux [3].

\section{Analysis of structure $\left(k_{1}\right)$, structure $\left(k_{2}\right)$ and structure $\left(k_{1} k_{2}\right)$ designs for two way elimination}

Consider a block design with $v$ treatments in $b$ blocks such that each block contains $k$ experimental units and each treatment is replicated $r$ times. If we consider the design as an array with $k$ rows and $b$ columns where the entries in the array consist of the treatment numbers, the analysis of structure (k) designs follows from the work of Zelen and Federer [19]. Define the matrices $N=\left(n_{i j}\right)$ and $\tilde{N}=\left(\tilde{n}_{i h}\right)$ of dimensions $v \times b$ and $v \times k$ respectively, where $n_{i j}=$ number of times treatment $i$ occurs in block $j$ and $\tilde{n}_{i h}=$ number of times treatment $i$ occurs in row $h$. The matrix $N$ is the incidence matrix for the design which relates the treatments to the (columns) blocks. We call $N$ as the column incidence matrix and $\tilde{N}$ as the row incidence matrix. Using the matrices $N$ and $\tilde{N}$, we can also define column $C$-matrix and row $C$-matrix. The column $C$-matrix is defined by

$$
C=R-N K^{-1} N^{\prime}
$$

where, $R=\operatorname{diag}\left(r_{1}, r_{2}, \ldots, r_{v}\right)$, and $K=\operatorname{diag}\left(k_{1}, k_{2}, \ldots, k_{b}\right)$.

The row $C$-matrix is defined by

$$
\tilde{C}=\tilde{R}-\tilde{N} \tilde{K}^{-1} \tilde{N}^{\prime}
$$

where, $\tilde{R}=\operatorname{diag}\left(\tilde{r}_{1}, \tilde{r}_{2}, \ldots, \tilde{r}_{v}\right)=R$ and $\tilde{K}=\operatorname{diag}\left(\tilde{k}_{1}, \tilde{k}_{2}, \ldots, \tilde{k}_{k}\right)$.

Let $Y_{j h}(j=1,2, \ldots, b ; h=1,2, \ldots, k)$ denote the measurement made in the $j$ th block and $h$ th row. When treatment $i$ is in block $j$ and row $h$, the random variable $Y_{j h}$ is assumed to have expected value $E\left(Y_{j h}\right)=\mu+\tau_{i}+b_{j}+r_{h}$, where $\mu$ is a constant, and $\tau_{i}, b_{j}$, and $r_{h}$ are fixed effects associated with the treatments, blocks and rows respectively. These parameters satisfy the constraints $\sum_{i=1}^{v} \tau_{i}=$ $\sum_{j=1}^{b} b_{j}=\sum_{h=1}^{k} r_{h}=0$. We assume that $Y_{j h}$ 's are uncorrelated with common variance $\sigma^{2}$.

When we analyze such design, our interest is usually focussed on estimating the treatment effect $\tau_{i}$. The estimates of the treatment effects can be obtained by solving a set of $v$ simultaneous linear equations which depend on the incidence matrices $N$ and $\tilde{N}$, and the adjusted treatment totals, which are functions of the observations. The adjusted treatment total for $i$ th treatment is given by

$$
Q_{i}=T_{i}-\sum_{j=1}^{b}\left(n_{i j} B_{j}\right) / k-\sum_{h=1}^{k}\left(\tilde{n}_{i h} R_{h}\right) / b+G / v
$$

where $T_{i}=$ total for treatment $i$,

$$
\begin{aligned}
& B_{j}=\sum_{h=1}^{k} Y_{j h}=\text { total for } j \text { th block, } \\
& R_{h}=\sum_{j=1}^{b} Y_{j h}=\text { total for } h \text { th row, and }
\end{aligned}
$$




$$
G=\sum_{i=1}^{v} T_{i}=\sum_{j=1}^{b} B_{j}=\sum_{h=1}^{k} R_{h}=\sum_{j=1}^{b} \sum_{h=1}^{k} Y_{j h} .
$$

The adjusted treatment totals $Q_{i}$ 's in (3.1) can be expressed as

$$
Q=T-\frac{1}{k}(N B)-\frac{1}{b}(\tilde{N} R)+\frac{G}{v} \mathbf{1}
$$

where $T_{(v \times 1)}, B_{(b \times 1)}$, and $R_{(k \times 1)}$ are the column vectors of the treatment, block and row totals respectively, and 1 denotes a $v \times 1$ vector of 1 's.

Now using Tochar [16], the reduced normal equations for estimating the treatment effect vector $\tau^{\prime}=\left(\tau_{1}, \tau_{2}, \ldots, \tau_{v}\right)$ can be written as

$$
Q=\left[r I-\frac{1}{k}\left(N N^{\prime}\right)-\frac{1}{b}\left(\tilde{N} \tilde{N}^{\prime}\right)+\left(\frac{r}{v}\right) \mathbf{1 1}^{\prime}\right] \hat{\tau}
$$

where $I$ is an identity matrix of order $v$. The estimate of the variance is

$$
S^{2}=\left[Y^{\prime} Y-\hat{\tau}^{\prime} Q-\frac{1}{b}\left(R^{\prime} R\right)-\frac{1}{k}\left(B^{\prime} B\right)+\frac{G^{2}}{v r}\left(\mathbf{1}^{\prime} \mathbf{1}\right)\right] / v_{e}
$$

where $v_{e}=(b k-b-v-k+2)$, the degrees of freedom of $S^{2}$.

\section{Structure $\left(k_{1}\right)$, structure $\left(k_{2}\right)$ and structure $\left(k_{1} k_{2}\right)$ properties and factorial structure}

As mentioned earlier, a structural property of a design which is related to the block incidence matrix or column $C$-matrix of the design, is given by

$$
N N^{\prime}=\sum_{j=1}^{w} \xi_{j} R_{j}
$$

where $w$ is some positive integer, $\xi_{1}, \xi_{2}, \ldots, \xi_{w}$ are some numbers, $R_{j}=\left(R_{j 1} \otimes R_{j 2} \otimes \ldots \otimes R_{j m}\right)$ and for each $j, R_{j i}$ is a $s_{i} \times s_{i}$ permutation matrix. This structural property is termed as structure $(k)$.

In the present work we call it a structure $\left(k_{1}\right)$ property. We can define a similar property for the row incidence matrix $\tilde{N}$ and call it a structure $\left(k_{2}\right)$ property which is given by

$$
\tilde{N} \tilde{N}^{\prime}=\sum_{j=1}^{w} \tilde{\xi}_{j} R_{j}
$$

where $\tilde{\xi}_{1}, \tilde{\xi}_{2}, \ldots, \tilde{\xi}_{w}$ are some numbers.

When the structure properties $\left(k_{1}\right)$ and $\left(k_{2}\right)$ both hold, we have

$$
\left[r I-\frac{1}{k}\left(N N^{\prime}\right)-\frac{1}{b}\left(\tilde{N} \tilde{N}^{\prime}\right)+\left(\begin{array}{l}
r \\
v
\end{array}\right) \mathbf{1 1}^{\prime}\right]=\sum_{j=1}^{w} \psi_{j} R_{j}
$$

for some numbers $\psi_{1}, \psi_{2}, \ldots, \psi_{w}$. 
We call this a structure $\left(k_{1} k_{2}\right)$ property. Substituting (4.3) in (3.3), we can write the reduced normal equations as given by

$$
\left(\sum_{j=1}^{w} \psi_{j} R_{j}\right) \hat{\tau}=Q
$$

that is,

$$
D \hat{\tau}=Q
$$

where $D=\sum_{j=1}^{w} \psi_{j} R_{j}$.

On this basis we can define structure $\left(k_{1}\right)$, structure $\left(k_{2}\right)$ and structure $\left(k_{1} k_{2}\right)$ properties as follows.

Definition 4.1. Structure $\left(k_{1}\right)$ property: If a column $C$-matrix of connected block design satisfies the relation (4.1), then it is called a structure $\left(k_{1}\right)$ property.

Definition 4.2. Structure $\left(k_{2}\right)$ property: If a row $C$-matrix of connected block design satisfies the relation (4.2), then it is called a structure $\left(k_{2}\right)$ property.

Definition 4.3. Structure $\left(k_{1} k_{2}\right)$ property: If the column and row $C$-matrices satisfy the relation (4.3), then it is called a structure $\left(k_{1} k_{2}\right)$ property.

Remark 1. From the definitions $4.1-4.3$, it is clear that structure $\left(k_{1}\right)$ and structure $\left(k_{2}\right)$ implies structure $\left(k_{1} k_{2}\right)$ property.

Remark 2. From definitions $4.1-4.3$, one can see that property $(A)$, property $(B)$ and property $(A B)$ are special cases of structure $\left(k_{1}\right)$, structure $\left(k_{2}\right)$ and structure $\left(k_{1} k_{2}\right)$ respectively.

Mukerjee [10] has shown that a necessary and sufficient condition for factorial structure in connected block design is that $C$-matrix has structure $(k)$ property. Interestingly, in the present work, we observe that a necessary and sufficient condition for factorial structure is that column $C$-matrix has structure $\left(k_{1}\right)$ or row $C$-matrix has structure $\left(k_{2}\right)$ or column and row $C$-matrices have structure $\left(k_{1} k_{2}\right)$ property.

Theorem 4.1. For a connected block design, a necessary and sufficient condition for factorial structure is that $C$-matrix has structure $\left(k_{1}\right)$.

Proof. The proof follows from Lemma 2.3.

Theorem 4.2. For a $v \times v$ permutation matrix $R$ and for any $x, W^{x} R W^{x}$ has structure $\left(k_{2}\right)$. Proof. The proof follows from Lemma 2.2.

Theorem 4.3. For a connected block design, a necessary and sufficient condition for factorial structure is that row $C$-matrix has structure $\left(k_{2}\right)$ property.

Proof. The proof follows from Theorem 4.2 and Lemma 2.3.

For a connected, equi-replicate and proper block design, factorial structure holds if and only if column incidence matrix has structure $\left(k_{1}\right)$ or row incidence matrix has structure $\left(k_{2}\right)$ or row and column incidence matrices have structure $\left(k_{1} k_{2}\right)$. 


\section{An example}

Consider a hypercubic design (HCD) with parameters $v=2^{2}, b=4, r=2, k=2, \lambda_{1}=1$ and $\lambda_{2}=0$ whose blocks are

The structures of row-column designs are

$$
\begin{gathered}
N N^{\prime}=\left(\begin{array}{ll}
N_{1} & N_{2} \\
N_{2} & N_{1}
\end{array}\right), \quad \tilde{N} \tilde{N}^{\prime}=\left(\begin{array}{ll}
\tilde{N}_{1} & \tilde{N}_{2} \\
\tilde{N}_{3} & \tilde{N}_{4}
\end{array}\right), \\
C=\left(\begin{array}{ll}
C_{1} & C_{2} \\
C_{2} & C_{1}
\end{array}\right), \quad \tilde{C}=\left(\begin{array}{ll}
\tilde{C}_{1} & \tilde{C}_{2} \\
\tilde{C}_{3} & \tilde{C}_{4}
\end{array}\right) .
\end{gathered}
$$

In this example,

$$
N N^{\prime}=\left(\begin{array}{llll}
2 & 1 & 1 & 0 \\
1 & 2 & 0 & 1 \\
1 & 0 & 2 & 1 \\
0 & 1 & 1 & 2
\end{array}\right)
$$

Now, we can write $N N^{\prime}$ as given by

$$
\begin{aligned}
N N^{\prime} & =1\left\{\left(\begin{array}{ll}
1 & 0 \\
0 & 1
\end{array}\right) \otimes\left(\begin{array}{ll}
1 & 0 \\
0 & 1
\end{array}\right)\right\}+1\left\{\left(\begin{array}{ll}
1 & 0 \\
0 & 1
\end{array}\right) \otimes\left(\begin{array}{ll}
1 & 0 \\
0 & 1
\end{array}\right)\right\} \\
& +1\left\{\left(\begin{array}{ll}
1 & 0 \\
0 & 1
\end{array}\right) \otimes\left(\begin{array}{ll}
0 & 1 \\
1 & 0
\end{array}\right)\right\}+1\left\{\left(\begin{array}{ll}
0 & 1 \\
1 & 0
\end{array}\right) \otimes\left(\begin{array}{ll}
1 & 0 \\
0 & 1
\end{array}\right)\right\} \\
& =\left(\begin{array}{llll}
1 & 0 & 0 & 0 \\
0 & 1 & 0 & 0 \\
0 & 0 & 1 & 0 \\
0 & 0 & 0 & 1
\end{array}\right)+\left(\begin{array}{llll}
1 & 0 & 0 & 0 \\
0 & 1 & 0 & 0 \\
0 & 0 & 1 & 0 \\
0 & 0 & 0 & 1
\end{array}\right)+\left(\begin{array}{llll}
0 & 1 & 0 & 0 \\
1 & 0 & 0 & 0 \\
0 & 0 & 0 & 1 \\
0 & 0 & 1 & 0
\end{array}\right)+\left(\begin{array}{llll}
0 & 0 & 1 & 0 \\
0 & 0 & 0 & 1 \\
1 & 0 & 0 & 0 \\
0 & 1 & 0 & 0
\end{array}\right) \\
& =\sum_{j=1}^{4} \xi_{j}\left(R_{j 1} \otimes R_{j 2}\right)
\end{aligned}
$$

where $\xi_{1}=1, \xi_{2}=1, \xi_{3}=1$ and $\xi_{4}=1$ and $v=\prod_{i=1}^{2} s_{i}=4$. 
Also, the $C$-matrix can be written as

$$
\begin{aligned}
C & =\left(\begin{array}{cccc}
1 & -\frac{1}{2} & -\frac{1}{2} & 0 \\
-\frac{1}{2} & 1 & 0 & -\frac{1}{2} \\
-\frac{1}{2} & 0 & 1 & -\frac{1}{2} \\
0 & -\frac{1}{2}-\frac{1}{2} & 1
\end{array}\right) \\
& =\frac{1}{2}\left\{\left(\begin{array}{ll}
1 & 0 \\
0 & 1
\end{array}\right) \otimes\left(\begin{array}{ll}
1 & 0 \\
0 & 1
\end{array}\right)\right\}+\frac{1}{2}\left\{\left(\begin{array}{ll}
1 & 0 \\
0 & 1
\end{array}\right) \otimes\left(\begin{array}{ll}
1 & 0 \\
0 & 1
\end{array}\right)\right\} \\
& +\left(-\frac{1}{2}\right)\left\{\left(\begin{array}{ll}
1 & 0 \\
0 & 1
\end{array}\right) \otimes\left(\begin{array}{ll}
0 & 1 \\
1 & 0
\end{array}\right)\right\}+\left(-\frac{1}{2}\right)\left\{\left(\begin{array}{ll}
0 & 1 \\
1 & 0
\end{array}\right) \otimes\left(\begin{array}{ll}
1 & 0 \\
0 & 1
\end{array}\right)\right\} \\
& =\sum_{j=1}^{4} \xi_{j}\left(R_{j 1} \otimes R_{j 2}\right)
\end{aligned}
$$

where $\xi_{1}=\frac{1}{2}, \xi_{2}=\frac{1}{2}, \xi_{3}=-\frac{1}{2}$ and $\xi_{4}=-\frac{1}{2}$.

Hence the above hypercubic design possesses structure $\left(k_{1}\right)$ property. In a similar way, we can express $\tilde{N} \tilde{N}^{\prime}$ and $\tilde{C}$ as linear combinations of Kronecker products of proper matrices of order $s_{1}$ and $s_{2}$. Now, it is clear that the hypercubic design possesses structure $\left(k_{2}\right)$ and structure $\left(k_{1} k_{2}\right)$ properties. Note that it also holds the property that structure $\left(k_{1}\right)$ and structure $\left(k_{2}\right)$ implies structure $\left(k_{1} k_{2}\right)$.

\section{Optimal R-type structure $(k)$ designs}

We will use $0,1, \ldots, v$ to denote the $(v+1)$ treatments being studied, with 0 representing the control treatment and $1,2, \ldots, v$ representing the test treatments. In this section, we consider those designs that have equal block sizes for comparing several test treatments with a control. Assuming that homoscedasticity is satisfied, we study these designs with respect to the trace, $A$ - and $M V$-optimality criteria.

Here we will use $d\left(v^{\prime}, b, k^{\prime}\right)$ to denote some particular block design that can be used in an experimental setting. The structure $(k)$ design in the previous example is not optimal within the test treatments. However, if we augment one control in each block of such design, then we see that structure $(k)$ design discussed in the example is optimal in the test treatment versus control treatment. It is interesting to see that augmented structure $(k)$ design satisfy trace, $A$ - and $M V$-optimality criteria. Here the augmented structure $(k)$ design also satisfy the condition $r_{0}=b$, that is, replication of the control treatment is equal to number of blocks of the design $d$. So we call it an optimal $R$-type structure $(k)$ design. Thus, using the previous example, a $R$-type structure $(k)$ design can be obtained as

In this example, we see that $v=4, b=4, k^{\prime}=3, r_{0}=4, m=2, n=2, \lambda_{0}=2, \lambda_{1}=0, \lambda_{2}=1$, $\operatorname{tr}_{d 11}^{-1}=3.5$,

$$
\begin{gathered}
m_{1}(4)=b(k-1) / v k=0.666666 \\
m_{2}(4)=\left\{\left(A-m_{1}(4)\right)-[(v-1) /(v-2)]^{1 / 2} P_{1}\right\} /(v-1)=(4.666667-0.666667) / 3=1.33334
\end{gathered}
$$


where

$$
\begin{gathered}
A=b(k-1)^{2} / k=16 / 3=5.333333 . \\
P_{1}=\left[\left(B-\left(m_{1}(4)\right)^{2}\right)-\left(A-m_{1}(4)\right)^{2} /(v-1)\right]^{1 / 2}=[7.555556-7.259260]^{1 / 2}=0.544331 \\
B=\operatorname{tr} C_{d 11}^{2}=8
\end{gathered}
$$

and

where

$$
H_{2}(4)=\left(1 / m_{1}(4)\right)+\left((v-1) / m_{4}(4)\right)=1.500002+2.25001=3.750003
$$

$$
m_{4}(4)=\left\{A-(2 / k)-m_{1}(4)\right\} /(v-1)=\left\{5.333333-\frac{2}{3}-0.666666\right\} / 3=1.333333 .
$$

Here $C_{d}=\operatorname{diag}\left(r_{d 0}, \ldots, r_{d v}\right)-\frac{1}{k} N_{d} N_{d}^{\prime}$ where $\operatorname{diag}\left(r_{d 0}, \ldots, r_{d v}\right)$ denotes a $(v+1) \times(v+1)$ diagonal matrix and the $i^{t h}$ row sum of $N_{d}$ is denoted by $r_{d i}$ which represents the number of times treatment $i$ is replicated in the design. The matrix $C_{d}$ is called the $C$-matrix of design $d$ and is positive semi-definite with zero row sums. In the above example, the incidence matrix and $C$ matrix of design $d$ are

$$
\begin{aligned}
& N_{d}=\left(\begin{array}{llll}
1 & 1 & 1 & 1 \\
1 & 0 & 1 & 0 \\
0 & 1 & 1 & 0 \\
1 & 0 & 0 & 1 \\
0 & 1 & 0 & 1
\end{array}\right) \\
& N_{d} N_{d}^{\prime}=\left(\begin{array}{lllll}
4 & 2 & 2 & 2 & 2 \\
2 & 2 & 1 & 1 & 0 \\
2 & 1 & 2 & 0 & 1 \\
2 & 1 & 0 & 2 & 1 \\
2 & 0 & 1 & 1 & 2
\end{array}\right) \\
& C_{d}=\left(\begin{array}{ccccc}
\frac{8}{3} & -\frac{2}{3} & -\frac{2}{3} & -\frac{2}{3} & -\frac{2}{3} \\
-\frac{2}{3} & \frac{4}{3} & -\frac{1}{3} & -\frac{1}{3} & 0 \\
-\frac{2}{3} & -\frac{1}{3} & \frac{4}{3} & 0 & -\frac{1}{3} \\
-\frac{2}{3} & -\frac{1}{3} & 0 & \frac{4}{3} & -\frac{1}{3} \\
-\frac{2}{3} & 0 & -\frac{1}{3} & -\frac{1}{3} & \frac{4}{3}
\end{array}\right) .
\end{aligned}
$$

Now we need to verify that the above structure $(k)$ design satisfies trace, $A$ - and $M V$-optimality criteria. Here we see that control treatment is replicated once in each block. So this is an SR(1) design. It is also obvious from the above example that in the original design all blocks have the same size, all the test treatments are replicated same number of times in blocks and $v=m n=2 \times 2=4$ treatments are divided into 2 disjoint sets of size 2 such that the treatment in the same group occur in $\lambda_{1}=0$ blocks together whereas treatment occurring in different groups occur in $\lambda_{2}=1$ blocks together. So it is a Group Divisible Design. Clearly, the design $d$ satisfies all the conditions of Lemma 2.5 and hence the design $d$ is trace optimal.

We will now show that the design $d$ constructed in the above example is $A$-optimal. 
From the above structure $(k)$ design, we observe that

$$
\begin{gathered}
m_{1}(4)=0.666666<m_{2}(4)=1.33334 \\
\operatorname{tr}_{d 11}^{-1}=3.5<H_{2}(4)=3.750003
\end{gathered}
$$

where $C_{d 11}$ is the principal submatrix obtained from $C_{d}$ after deleting row 1 and column 1 . So the design $d$ satisfies all the conditions of Theorem 2.4 and hence the above structure $(k)$ design is A-optimal.

We will now show that the design $d$ is $M V$-optimal. We first calculate the following:

$$
\begin{gathered}
N\left(r_{0}\right)=\left[\frac{r_{0}}{b}\right]=1 \\
\lambda\left(r_{0}\right)=\left(r_{0}-b N\left(r_{0}\right)\right)\left(N\left(r_{0}\right)+1\right)^{2}+\left(b-r_{0}+b N\left(r_{0}\right)\right) N^{2}\left(r_{0}\right)=4 \\
\left.R\left(r_{0}\right)=\left[\left(b k-r_{0}\right) / v\right)\right]=\frac{8}{4}=2 \\
r_{0} k-\lambda\left(r_{0}\right)=12-4=8 \\
\bar{\lambda}_{0}=\left(r_{0} k-\lambda\left(r_{0}\right)\right) / v=2, \bar{\lambda}_{1}=\left[\left(R\left(r_{0}\right)(k-1)-\bar{\lambda}_{0}\right) /(v-1)\right]=[0.7]=0, \bar{\lambda}_{2}=\bar{\lambda}_{1}+1=1 \\
\bar{m} k=\left(k / v \bar{\lambda}_{0}\right)+\left((\bar{v}-1) s k / v\left(\bar{v}(s-1) \bar{\lambda}_{2}+v \bar{\lambda}_{1}+\bar{\lambda}_{0}\right)\right)+\left((s-1) k / v\left(v \bar{\lambda}_{2}+\bar{\lambda}_{0}\right)\right)=\frac{7}{8}=0.875 \text { with } \\
\bar{v}=v / s=2 . \\
\bar{c}_{d 00}=\left(r_{0} k-\lambda\left(r_{0}\right)\right) / k=2.666667 \\
\bar{c}_{d i i}=R\left(r_{0}\right)(k-1) / k=1.333333 \\
\bar{c}_{d i o}=-\left(\bar{\lambda}_{0}-1\right) / k=-0.333333 \\
\bar{c}_{d j j}=\left(R\left(r_{0}\right)(k-1)-2\right) / 2=0.666667 \\
\bar{c}_{d j 0}=-\bar{\lambda}_{0} / k=-0.666667 \\
\bar{c}_{d p p}=\bar{c}_{d q q}=R\left(r_{0}\right)(k-1) / k=1.333333 \\
\bar{c}_{d p 0}=\bar{c}_{d q 0}=-\bar{\lambda}_{0} / k=-0.666667 \\
-\bar{c}_{d p q}=\left(\bar{\lambda}_{1}-1\right) / k=-0.333333 .
\end{gathered}
$$

Now we verify the four conditions of Theorem 2.5.

(1) $r_{0} k-\lambda\left(r_{0}\right)-2=8-2=6<v B\left(r_{0} k-\lambda\left(r_{0}\right)-2, R\left(r_{0}\right)(k-1)\right)=8.8625$

(2) $1 /\left(R\left(r_{0}\right)-1\right)(k-1)=0.5>\bar{m}=0.2917$

(3) $\bar{m} k=0.875<\min \left\{\left(\bar{c}_{d 00}+\bar{c}_{d i i}+2 \bar{c}_{d i 0}\right) /\left(\bar{c}_{d 00} \bar{c}_{d i i}-\bar{c}_{d i o}^{2}\right),\left(\bar{c}_{d 00}+\bar{c}_{d j j}+2 \bar{c}_{d j 0}\right) /\left(\bar{c}_{d 00} \bar{c}_{d j j}-\right.\right.$ $\left.\left.\bar{c}_{d j 0}^{2}\right)\right\}=0.967742$

(4) $\bar{m} k=0.875<\left\{\bar{c}_{d p p}\left(\bar{c}_{d 00}+\bar{c}_{d q q}+2 \bar{c}_{d p q}\right)-\left(\bar{c}_{d p 0}+\bar{c}_{d p q}\right)^{2}\right\} /\left\{\bar{c}_{d 00} \bar{c}_{d p p} \bar{c}_{d q q}-\bar{c}_{d 00} \bar{c}_{d p q}^{2}-\right.$ $\left.\bar{c}_{d p p} \bar{c}_{d q 0}^{2}-\bar{c}_{d q q} \bar{c}_{d p 0}^{2}+2 \bar{c}_{d p 0} \bar{c}_{d q 0} \bar{c}_{d p q}\right\}=1.145833$

We see that the conditions given in Theorem 2.5 are verified. Thus, the above structure $(k)$ design is $M V$-optimal. Hence we conclude that the design $d(5,4,3)$ is trace, $A$ - and $M V$-optimal design. 


\section{Acknowledgments}

The authors sincerely thank the editor and the referees for their helpful comments and suggestions that have resulted in an improved version of this manuscript. The research of S. Mandal is supported by a Discovery Grant from the Natural Sciences and Engineering Research Council (NSERC) of Canada.

\section{References}

[1] S. C. Cotter, Confounding in Factorial Experiment, Unpublished Ph.D.Thesis (University of Southamption, U.K., 1973).

[2] M. Jacroux, The A-optimality block designs for comparing test treatments with a control, Journal of the American Statistical Association, 84 (1989), 310-317.

[3] M. Jacroux, On the determination and construction of $M V$-optimal designs for comparing test treatments with a standard treatment, Journal of Statistical Planning and Inference, 15 (1987), 205-225.

[4] M. Jacroux, On the optimality of block designs for comparing test treatments with a control, Technical Report, Dept. of Mathematical Sciences, Washington State University, (1987).

[5] M. Jacroux, On the optimality and usage of reinforced block designs for comparing test treatments with a standard treatment, Journal of the Royal Statistical Society B, 46 (1984), 316-322.

[6] J. A. John and T. M. F. Smith, Two-factor experiments in non-orthogonal designs, Journal of the Royal Statistical Society B, 34 (1972), 401-409.

[7] B. M. Kurkjian and M. Zelen, Application of the calculus of factorial arrangements: Block and direct product designs, Biometrika, 50 (1963), 63-73.

[8] B. M. Kurkjian and M. Zelen, A calculus for factorial arrangements, Annals of Mathematical Statistics, 33 (1962), 600-619.

[9] R. Mukerjee, Further results on the analysis of factorial experiments, Calcutta Statistical Association Bulletin, 29 (1980), 1-26.

[10] R. Mukerjee, Inter-effect orthogonality in factorial experiments, Calcutta Statistical Association Bulletin, 28 (1979), 83-108.

[11] V. B. Paik and W. T. Federer, Partially balanced design act properties A and B, Communications in Statistics, 1 (1973), 331-350.

[12] P. V. Rao, Analysis of a class PBIB designs with more than two associate classes, Annals of Mathematical Statistics, 32 (1961), 800-808.

[13] B. V. Shah, On a generalization of the Kronecker product designs, Annals of Mathematical Statistics, 30 (1959), 48-54.

[14] L. L. Sia, Optimum spacing of elementary treatment contrasts in symmetrical 2-factor PA block Designs, Canadian Journal of Statistics, 5 (1977), 227-234.

[15] A. Thannipara, On optimal block designs for comparing test treatments with a control, Unpublished Ph.D. thesis (Saurashtra University, Rajkot, India, 1992).

[16] K. D. Tocher, The design and analysis of block experiments (with discussion), Journal of the Royal Statistical Society B, 14 (1952), 45-100.

[17] M. N. Vartak, On an application of Kronecker product of matrices to statistical designs, Annals of Mathematical Statistics, 26 (1955), 420-438.

[18] M. Zelen and W. T. Federer, Applications of the calculus for factorial arrangements III: Analysis of factorials with unequal number of observations, Sankhyā A, 27 (1965), 383-400.

[19] M. Zelen and W. T. Federer, Applications of the Calculus for factorial arrangements II: Two way elimination of heterogeneity, Annals of Mathematical Statistics, 35 (1964), 658-672. 